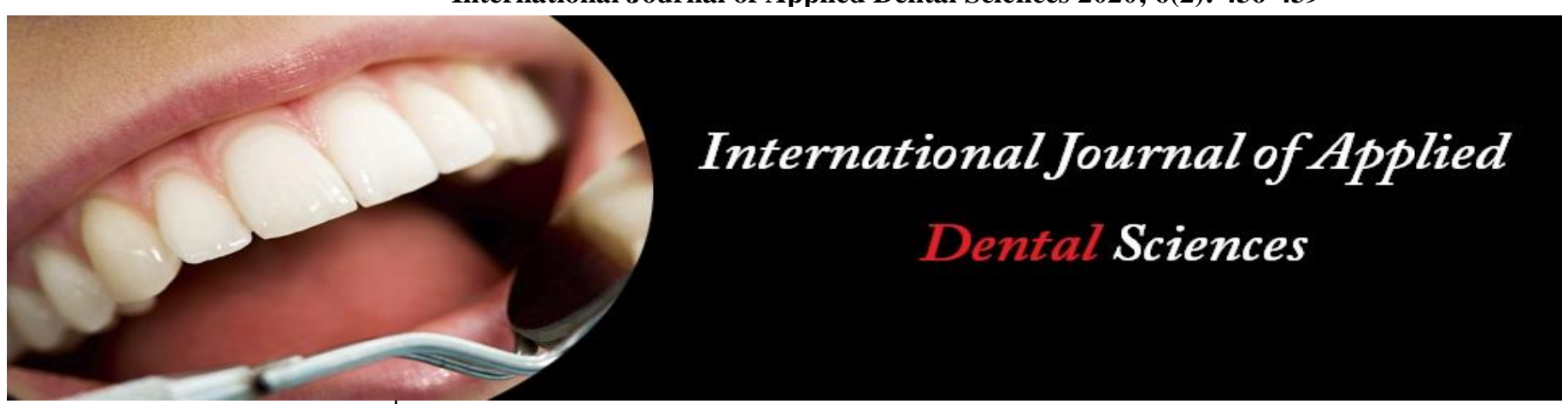

ISSN Print: 2394-7489

ISSN Online: 2394-7497

IJADS 2020; 6(2): 436-439

(C) 2020 IJADS

www.oraljournal.com

Received: 13-02-2020

Accepted: 17-03-2020

Abhimanyu Rohmetra

Senior Lecturer,

Department of Orthodontics and

Dentofacial Orthopaedics,

Institute of Dental Sciences,

Sehora, Jammu, J\&K. India

Niharika Gupta

PG Student,

Department of Oral and

Maxillofacial Surgery,

J.N. Kapoor DAV(C) Dental

College, Yamunanagar,

Haryana, India

Ishita

Intern, Institute of Dental

Sciences, Sehora, Jammu,

J\&K, India

\section{Comparison of two orthodontic indirect bonding methods- light cure vs chemical cure}

\author{
Abhimanyu Rohmetra, Niharika Gupta and Ishita
}

DOI: https://doi.org/10.22271/oral.2020.v6.i2g.888

\section{Abstract}

Introduction: The study was undertaken to compare two indirect bonding methods, one method used a light cured adhesive system and clear vacuum formed transfer trays and a second method used a chemical cure adhesive system and putty transfer trays

Materials and Methods: 191 bondings provided a data base of bond failures for two indirect bonding methods. The initial bond failure rates were compared using Fisher's Exact Test. The long term bond failure rates were compared using actuarial life tables and a Mantel-Haenzel comparison.

Results: In initial bond failure the light cured method had a $9 \%$ failure rate compared to the chemical cured methods $0.7 \%$ failure rate. Long term the light cured method had a $0 \%$ failure rate while the chemical cured method had a $9 \%$ failure rate.

Conclusion: The initial bond was less likely to fail with the chemically cured method but long term the light cured method was had fewer bond failures.

Keywords: orthodontic, light cure vs chemical cure

\section{Introduction}

When bonding fixed orthodontic appliances to teeth orthodontists have the choice of bonding indirectly or directly. Indirect bonding involves positioning the brackets on models of the teeth and then transferring the brackets to the patient's mouth to be bonded. This allows for determining the position of the bracket in the lab away from oral sources of contamination. It also allows for the evaluation of bracket position without the use of a mirror and from view points that would be difficult or impossible if positioning brackets directly in the mouth ${ }^{[1]}$.

Once the bracket position is satisfactory a transfer tray is fabricated and the brackets can then be transferred to the patient's mouth with all of the brackets maintaining the chosen position. Conversely direct bonding involves positioning the brackets in the mouth and then bonding the bracket in place. This allows more time for the adhesive system used to bond the bracket to become contaminated ${ }^{[2]}$. Also, due to confined environment on the mouth often times in is difficult to fully visualize the bracket position.

A large factor in initial and long term bond failure is contamination of the bonding site by saliva, blood, and or water. This contamination can occur after etching or after the placement of the primer/bonding agent. In the light cured indirect bonding method being studied brackets are placed in the mouth in groups of 5-12 and then bonded in place individually. The light cured method recommends a total of 10 seconds of curing per tooth in two separate 5 second intervals. The chemically cured indirect bonding method being studied places the brackets in the mouth in groups of 5-12 which are bonded simultaneously to the teeth. The chemical cure requires a 4 minute set time. The difference in timing and manner of curing allow for separate paths of contamination with no clear advantage to either method.

Bond failures can represent a significant increase in treatment time. In 2007, a study by Haeger et al. ${ }^{[3]}$ found an average increase of 1.21 months of treatment time per bond failure. Typical treatment times range from 16-24 months with each bond failure representing a 5-8\% potential increase in treatment time. Longer treatment time increases the risk of root resorption and carious white spot lesions. Root resorption decreases the amount of tooth structure supported by bone and compromises the long term health of the tooth. White spot lesions are precursors
Corresponding Author: Abhimanyu Rohmetra Senior Lecturer, Department of Orthodontics and Dentofacial Orthopaedics, Institute of Dental Sciences, Sehora, Jammu, J\&K, India 
To dental cavities and also present an aesthetic blemish on the tooth that is permanent.

By determining if certain indirect bonding methods provide for decreased bond failure procedures could be selected that would reduce overall orthodontic treatment time. This would decrease the risk of root resorption and white spot lesions. ${ }^{[4]}$ This study compared the bond failure of two indirect bonding methods to evaluate their effectiveness at creating clinically sufficient bonds. The aims of this study were to show if one bonding method had fewer initial and long term bond failures. The bonding methods evaluated were a light cured method that used a clear vacuum formed transfer tray and a chemically cured method that used putty transfer tray.

\section{Methods and Materials}

Orthodontic brackets adhere to teeth with dental composite resins. This can be done directly in the mouth or indirectly in a lab and then transferred to the mouth. In the indirect setup brackets with composite are positioned on patient models and the composite is cured. A transfer tray is then made to transfer the brackets from the models to the patient's mouth. In the mouth the bracket and composite assembly are adhered to the teeth using either a chemically polymerized dental adhesive or a light polymerized dental adhesive.

For this study one light cured method and one chemical cured method were evaluated. The steps necessary to perform each method follow a similar pattern and vary in only three key areas. These steps can be broken down into: an initial appointment, lab preparation, and a bonding appointment. ${ }^{[5,6]}$ At the initial appointment accurate impressions of the teeth are made. In the dental lab the impressions are poured in
White orthodontic stone. The dental models are then dried and coated with a separating medium. Once that is dry the brackets are placed and excess resin is removed. The brackets and resin are then light cured creating a custom resin pad on the back of the bracket that precisely fits the contours of each individual tooth. At this stage the transfer trays are made and the lab portion is complete. At the bonding appointment the teeth are cleaned and etched with $37 \%$ phosphoric acid for 30 seconds. Then the bonding system is place on the teeth and the brackets and the transfer tray with the brackets is placed in the patient's mouth. After the bonding is complete any excess bonding material is removed and treatment can begin.

The first area where the two methods vary is in the type of transfer tray that is fabricated. In the light cured method two clear trays are vacuum formed over the dental models and brackets. In the chemically cured method PVS putty is used and hand formed onto the dental models and brackets. ${ }^{\text {[7] }}$

The second area of difference is the manner in which the separating medium is removed from the back of the custom resin pad. In the light cured method the separating medium is removed by micro air abrasion with 50 micron alumina. In the chemically cured method the separating medium is removed with a scaler or other sharp instrument. ${ }^{[8]}$

The third difference is the bonding system used to adhere the brackets to the teeth. In the light cured method the bonding system uses Proseal on the teeth followed by Assure with Flowtain placed on the back of the custom resin pad. These three chemicals are all polymerized through light activated free radical polymerization. In the chemically cured method the tooth and the back of the custom resin pad is coated with Maximum Cure sealant. ${ }^{[9]}$

Table 1: Materials used in each bonding method

\begin{tabular}{|c|c|}
\hline Light cured method & Chemical cured method \\
\hline $\begin{array}{l}\text { - Alginate (imprEssix, Raintree Essix) } \\
\text { - Stone (Whip Mix Orthodontic Model Stone) } \\
\text { - Separating medium (Liquid Foil, Great Lakes Orthodontics) } \\
\text { - Adhesive (APC Transbond XT, 3M/Unitek) } \\
\text { - Soft plastic tray (1.5mm Bioplast, Raintree Essix) } \\
\text { - Hard plastic tray (1 mm Biocryl, Raintree Essix) } \\
\text { - Air abraison (50 micron silicon) } \\
\text { - Flour Pumice } \\
\text { - Etch (Ultra Etch, Ultra Dent) } \\
\text { - Reliance proseal (Reliance Orthodontics) } \\
\text { - Reliance Assure (Reliance Orthodontics) } \\
\text { - Curing light (OrthoLux, 3M/Unitek) }\end{array}$ & $\begin{array}{l}\text { - Alginate (imprEssix, Raintree Essix) } \\
\text { - Stone (Whip Mix Orthodontic Model Stone) } \\
\text { - Separating medium (Liquid Foil, Great Lakes Orthodontics) } \\
\text { - Adhesive (APC Transbond XT, 3M/LJnitek) } \\
\text { - Putty (Aquasil Easy Mix Putty, Dentsply Caulk) } \\
\text { - Flour Pumice } \\
\text { - Etch (Ultra Etch, Ultra Dent) } \\
\text { - Sealant (Maximum Cure Unfilled, Reliance Orthodontics) }\end{array}$ \\
\hline
\end{tabular}

\section{Inclusion/Exclusion Criteria}

For inclusion into the study the patient needed to have been bonded with orthodontic brackets

1. It must have been noted that they were bonded using one of the two indirect bonding methods outlined below.

2. Differences in brackets and bonding materials were eliminated by assuring that only cases bonded with 3M/Unitek Victory series .022 slot APC brackets, Ultra Etch, Assure and Flowtain were included.

Patients were excluded if:

1. Their teeth were hypo/hyper calcified

2. Had fluorosis

3. Were bleaching their teeth within the last 2 weeks before bonding as all of these factors affect bond strength.

\section{Data Collection}

Data was collected on an excel spread sheet indicating the date and type of indirect bonding method used, which teeth were bonded and if any initial bond failure occurred. Any subsequent bond failures up to 6 months were also recorded. Only the first bond failure of each tooth was recorded because subsequent rebonds were accomplished using a direct method. The spread sheet included areas for each bond failure to be recorded and then the failures were aggregated by bonding technique. Initial bond failures were any bond failure that occurred at the bonding appointment. All subsequent bond failures up to 6 months of treatment time were recorded.

\section{Statistical Analysis}

Descriptive statistics of the sample data can be found in table 2. To compare the two bonding methods Fisher's Exact Test was performed. Fisher's Exact Test is a derivation of the ChiSquare Test that is used when any expected value is less than 5. Fischer's Exact Test assumes the following: that the population from which the sample data was taken had a normal distribution of initial bond failure, that the variables used are numerical, not ratios or percentages, that the 
variables are independent, and that the variables are categorical. The categories that the variables fit into were the type of bonding method used and if the bond failed or did not fail. Fisher's Exact Test tests the hypothesis that the variables are independent of each other. In the case of initial bond failure the test shows weather the type of bonding system used is independent of the number of initial bond failures. To determine if there is a relationship between the type of bonding method used and the number of bond failures the contingency coefficient was examined. This statistic measures the relation between two categorical variables. It's range is from 0 to 1 with 0 meaning complete independence (StatSofi 2007).

Table 2: Descriptive statistics of the sample data

\begin{tabular}{|c|c|c|}
\hline & Number & Percentage \\
\hline Number of Patients & 9 & $100 \%$ \\
\hline \multicolumn{3}{|c|}{ Number of Patients per bonding method } \\
\hline $\begin{array}{l}\text { Light cured } \\
\end{array}$ & 3 & $33 \%$ \\
\hline Chemically cured & 6 & $66 \%$ \\
\hline \multicolumn{3}{|c|}{ Distribution of patients by sex } \\
\hline Female & 3 & $33 \%$ \\
\hline Male & 6 & $66 \%$ \\
\hline \multicolumn{3}{|c|}{ Distribution of patients by age } \\
\hline 13 & 2 & $22 \%$ \\
\hline 14 & 3 & $33 \%$ \\
\hline 15 & 3 & $33 \%$ \\
\hline 29 & 1 & $11 \%$ \\
\hline Number of brackets & 191 & $100 \%$ \\
\hline \multicolumn{3}{|c|}{ Distribution of brackets by sex } \\
\hline Female & 54 & $28 \%$ \\
\hline Male & 137 & $72 \%$ \\
\hline \multicolumn{3}{|c|}{ Distribution of brackets by age } \\
\hline 13 & 36 & $19 \%$ \\
\hline 14 & 61 & $32 \%$ \\
\hline 15 & 70 & $37 \%$ \\
\hline 29 & 24 & $12 \%$ \\
\hline \multicolumn{3}{|c|}{ Distribution of brackets by tooth type } \\
\hline Upper incisors & 36 & $19 \%$ \\
\hline Lower incisors & 32 & $17 \%$ \\
\hline Upper canines & 18 & $9 \%$ \\
\hline Lower canines & 16 & $8 \%$ \\
\hline Upper premolars & 33 & $17 \%$ \\
\hline Lower premolars & 30 & $16 \%$ \\
\hline Upper molars & 14 & $7 \%$ \\
\hline Lower molars & 14 & $7 \%$ \\
\hline \multicolumn{3}{|c|}{$\begin{array}{l}\text { Distribution of brackets by bonding } \\
\text { method }\end{array}$} \\
\hline Putty/chemical cure & 135 & $71 \%$ \\
\hline Clear/light cure & 56 & $29 \%$ \\
\hline
\end{tabular}

To examine if one of the bonding systems created a longer lasting bond, data was collected on the number and location of bond failures from the time of bonding to 6 months afterwards. Any bond failures that occurred during the initial bonding appointment were not included. A survival analysis was performed to measure the time to bond failure. The survival analysis was done on an actuarial table bases with time interval of 180 days being the same for the entire sample. This analysis gave a 1 year projected survival rate for the two different bonding methods. Next a Mantel-Haenszel analysis was performed to compare the survival rates of the two bonding methods and determine if they were statistically different. The use of survival rate analysis was advocated by Miller $^{10}$ as an appropriate way to analyze bracket failure because it allows for a comparison between studies even when the time interval being evaluated varies. For this analysis we must assume that the bond failure is multiplicatively related to the time and group variables. This analysis generates a Chi-Square statistic and a corresponding $p$ value. The variable used was the length of the time the bracket was bonded, if the bracket bond failed during the 6 months and which group the bracket was associated with. If the bracket was still bonded at the end of 6 months then the value for the time variable was 180 days. The data was assumed to be from a normal distribution, the time variable was quantitative while the bond failure and group variables were categorical. All of the statistical analysis was performed using WINKS SDA software by TexaSoft.

\section{Results}

The frequency of initial bond failure for the putty/chemical cure method was 1 failed bonding in 135 attempts and for the clear/light cure method it was 4 failed bondings in 56 attempts. Table 3 details the bond failure sites for both initial bond failure and 6 month bond survival.

Table 4: Bond failure sites

\begin{tabular}{|c|c|c|}
\hline Bonding Method & Bracket & Failure timing \\
\hline Putty/chemical cure & LR2 & Initial bond failure \\
\hline & LR5 & Failed after 7 days \\
\hline & UL5 & Failed after 29 days \\
\hline & LL2 & Failed after 29 days \\
\hline & LL 1 & Failed after 29 days \\
\hline & LR5 & Failed after 30 days \\
\hline & LL 1 & Failed after 30 days \\
\hline & LL2 & Failed after 30 days \\
\hline & LL2 & Failed after 35 days \\
\hline & LR2 & Failed after 78 days \\
\hline & UL 1 & Failed after 137 days \\
\hline & LL3 & Failed after 137 days \\
\hline & LL4 & Failed after 137 days \\
\hline Clear/light cure & UR4 & Initial bond failure \\
\hline & UR5 & Initial bond failure \\
\hline & UR6 & Initial bond failure \\
\hline & UL5 & Initial bond failure \\
\hline & UL6 & Initial bond failure \\
\hline
\end{tabular}

Table 4 summarizes the comparison of initial bond failures for the two methods using Fisher's Exact Test. With a $p$ value of .009 we can have a high level of confidence that the results from the sample data are reflective of the sample population. To evaluate if there is a relationship between the bonding method and initial bond failure rates we find a contingency coefficient of 0.209. This can be interpreted as showing a weak or mild relationship between which bond method was used and the likelihood of a initial bond failure.

Table 4: Comparison of initial bond failure rates

\begin{tabular}{|c|c|c|c|c|c|}
\hline & Bond Failed & Bond did not fail & Total & Percent & $\boldsymbol{p}$ value \\
\hline Light cured & 5 & 51 & 56 & $0.7 \%$ & 0.009 \\
\hline Chemically cured & 1 & 134 & 135 & $9 \%$ & 0.009 \\
\hline Total & 6 & 185 & 191 & & \\
\hline
\end{tabular}


Table 5 summarizes the results of an actuarial life table for the survival rate of a bracket bonded with one of the two bonding methods and a corresponding $p$ value that indicates if the two survival rates are statistically different.

Table 5: Long term survival rate for the two bonding methods

\begin{tabular}{|c|c|c|c|c|}
\hline & Bond Failed & Bond did not fail & 360 day failure rate & $\boldsymbol{p}$ value \\
\hline Light cured & 0 & 51 & $0 \%$ & 0.03 \\
\hline Chemically cured & 12 & 122 & $9 \%$ & 0.03 \\
\hline Total & 12 & 173 & & \\
\hline
\end{tabular}

\section{Discussion}

The analysis shows that in this data set the putty/chemical cure method provided for fewer initial bond failures but the clear/light cure method provided for a longer lasting bond. Confounders to the analysis were: the type of tooth was not accounted for, the age and gender of the patient was not accounted for, three different operators were included in the data set, the exact day of bond failure was unknown and as a substitute the appointment day the failure was reported was the value used. The $p$ values found in this study were statistically significant. This indicates a small likelihood committing a Type 1 statistical error where the null hypothesis of both the bonding methods being equal is actually true when the study found it to be false. However because of the low power of the study, due to small sample size, there is a risk of committing a Type 2 statistical error where the null hypothesis is rejected when in fact it is true. The risk of committing this type of error could be reduced simply by increasing the sample size.

\section{Summary and Conclusion}

This study was designed to evaluate and compare the initial and long term bond failure rates of two orthodontic indirect bonding methods. The conclusions of this study are two fold: 1. The putty/chemical cure method results in fewer initial bond failures. This could be attributed to higher initial bond strength, the method could be less subject to operator error or the technique could allow for lower stress to the bracket bond system when the transfer tray is removed.

2. The clear/light cure method results in a bond that initially fails more but lasts longer. This could be attributed to low initial bond strength that build over time. Difficulty of the method on bonding day. High stress delivered to the bracket bond system when the clear trays are removed.

Attributing the bond failure to lack of bond strength is difficult. The manner of each bond failure is multivariable. This study does have some implications for the overall use of the two methods studied. The light cured method performed well over time and as such could result in fewer bond failures and less treatment time than the chemically cured method that had multiple bond failures after the initial bonding.

\section{References}

1. Bowen RL, Marjenhoff WA. Development of an adhesive bonding system. Oper Dent. 1962; 5(Suppl):7580 .

2. Newman GV. Bonding plastic orthodontic attachments to tooth enamel. J New Jersey State Dental Society 1964, 346-358.

3. Haeger RS, Colberg RT. Effects of missed appointments and bracket failures on treatment efficiency and office productivity. J Clin Orthod 2007; 41(8):433-437.

4. Newman GV, Snyder WH, Wilson MS, Hanesian D. Adhesives and orthodontic attachments. J New Jersey State Dental Society. 1965, 113-120.

5. Newman GV, Snyder WH. Wilson CEo Acrylic adhesives for bonding attachments to tooth surfaces. Angle Orthod 1968; 38(1):12-18.

6. Newman GV. Adhesion and orthodontic plastic attachments. Am J Orthod. 1969; 56(6):573-588.

7. Silverman E, Cohen M, Gianelly AA, Dietz VS. A universal direct bonding system for both metal and plastic brackets. Am J Orthod. 1972; 62(3):236-244.

8. Wisth PJ, Bergenkreutz K. Use of ultrasonic instruments in orthodontic practice. Angle Orthod. 1974; 44(3):251253.

9. Silverman E, Cohen M. Current adhesives for indirect bracket bonding. Am J Orthod. 1974; 65(1):76-84.

10. Miller JR. Commentary: basic concepts concerning bracket failure research. Angle Orthod. 1997; 67(3):167168. 